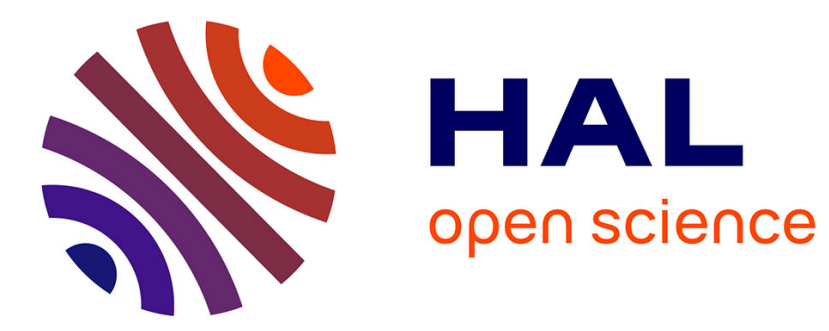

\title{
High spatial resolution detection of low-energy electrons using an event-counting method, application to point projection microscopy
}

Evelyne Salançon, Alain Degiovanni, Laurent Lapena, Roger Morin

\section{- To cite this version:}

Evelyne Salançon, Alain Degiovanni, Laurent Lapena, Roger Morin. High spatial resolution detection of low-energy electrons using an event-counting method, application to point projection microscopy. Review of Scientific Instruments, 2018, 89 (4), 10.1063/1.5020255 . hal-01784322

\section{HAL Id: hal-01784322 \\ https://hal.science/hal-01784322}

Submitted on 1 Feb 2019

HAL is a multi-disciplinary open access archive for the deposit and dissemination of scientific research documents, whether they are published or not. The documents may come from teaching and research institutions in France or abroad, or from public or private research centers.
L'archive ouverte pluridisciplinaire HAL, est destinée au dépôt et à la diffusion de documents scientifiques de niveau recherche, publiés ou non, émanant des établissements d'enseignement et de recherche français ou étrangers, des laboratoires publics ou privés. 
High spatial resolution detection of low-energy electrons using an event-counting method, application to point projection microscopy

Evelyne Salançon", Alain Degiovanni, Laurent Lapena and Roger Morin

Aix Marseille Univ, CNRS, CINAM, Marseille, France

\section{Abstract: An event-counting method using a two-microchannel plate stack in a low-energy electron point projection microscope is} implemented. $15 \mu \mathrm{m}$ screen-spatial resolution i.e. the distance between first-neighbor microchannels is demonstrated. This leads to a 7 times better microscope-resolution. Compared to previous work with neutrons ${ }^{1}$, the large number of detection events achieved with electrons shows that the local response of the detector is mainly governed by the angle between the hexagonal structures of the two microchannel plates. Using this method in point projection microscopy offers the prospect of working with a source-object distance one order of magnitude greater $(500 \mathrm{~nm}$ instead of $50 \mathrm{~nm})$, advancing toward atomic resolution.

\section{1- Introduction}

The fundamental spatial resolution of a microscope is given by Abbe's diffraction limit. This means that the smallest visible distance is given by $\varepsilon=\frac{\lambda}{2 N . A \text {. }}$, with $\lambda$ the wavelength of the wave used and N.A. the numerical aperture. The $N . A$. characterizes the angle over which the system detects waves propagating from the object. For a pure (lensless) projector, it is given by the geometry of the setup $N . A .=$ $R / \sqrt{D^{2}+R^{2}}$ where $\mathrm{R}$ is the radius of the detector and $D$ the object to screen distance. Thus, for a projector:

$\varepsilon=\frac{\lambda \sqrt{D^{2}+R^{2}}}{2 R}$
However in order to obtain this resolution $\varepsilon$, a suitable detector is required. Since a microscope locates absolute positions of a point in the object plane, a displacement of $\varepsilon$ in the object plane has to be detectable in the image plane. In that plane the corresponding displacement is $G \varepsilon$ where $\mathrm{G}$ is the geometrical magnification of the projector i.e. $G=\frac{D+d}{d}$, where $d$ is the source-object distance. Therefore the screen-spatial resolution $\eta$ required for the detector is $\eta=G \varepsilon$, thus:

$\varepsilon=\frac{\eta d}{D+d}$

Combining [1] and [2], we obtain for a projector:

$\varepsilon^{2}=\frac{\eta \lambda d \sqrt{D^{2}+R^{2}}}{2 R(D+d)}$

Or, for high enough magnification ( $D>>d)$ and $D \geq 2 R$ :

$d \approx\left(\frac{2 \varepsilon^{2}}{\lambda}\right) \cdot \frac{R}{\eta}$

This shows that for a detector of given screenresolution $\eta$ and radius $\mathrm{R}$ a microscoperesolution $\varepsilon$ (limited by Abbe's criteria) can always be obtained but at the cost of working at a small enough source-to-object distance $d$.

A low-energy electron point projection microscope (figure 1) is a projector based on an electron point source ${ }^{2}$. The image detector is usually a microchannel plate fluorescent screen assembly of radius $\mathrm{R}=20 \mathrm{~mm}$. The screenresolution $\eta$ of this assembly is about $100 \mu \mathrm{m}$ [see below and ${ }^{3}$ ]. Up to now, the microscopespatial resolution obtained with this setup has been about $1 \mathrm{~nm}^{4,5,6,{ }^{+}}$. In addition to angle limitations in the emission or in the scattering process and to noise problems (e.g. vibrations, parasitic electromagnetic fields) this is due to limitations inherent to the detector itself, especially in terms of its spatial resolution $\eta$. Equation [3'] shows that for $100 \mathrm{eV}$ electrons $(\lambda=1.22 \AA)$, a microscope-resolution of $\lambda$ can only be obtained for $d<50 \mathrm{~nm}$.

\footnotetext{
† Using a 75mm diameter detector, Fink's group at Zürich recently achieved $0.8 \mathrm{~nm}$ resolution on protein deposited on graphene.
}

* Corresponding author: Tel : +33660362805 / email adress : salancon@cinam.univ-mrs.fr 
Working at such short source-object distances, however, raises numerous problems with respect to high resolution imaging: (i) the field on the object increases with the decrease of $d$, causing image distortion ${ }^{7,8,9,10,11}$; (ii) decreasing $d$ induces a decrease in the emission voltage ${ }^{2}$, which impacts the resolution via both the increase in $\lambda$ and the blurring effect resulting from the electron energy spread; (iii) decreasing $d$ increases the influence of the finite size of the source ${ }^{12,13}$; (iv) the small $d$ approach prevents the use of promising sources ${ }^{14}$ more bulky than field emission tips, like the one used here.

An event-counting detection method using a microchannel plate (MCP) stack with a fluorescent screen and a CCD camera is already used for photon or neutron detection ${ }^{1,15,16,17}$ and shows sub- $15 \mu \mathrm{m}$ spatial resolution. The method is based on the fact that one single particle impact at the channel plate input creates one single stain on the screen. The centroid of this stain is attributed to the impact position of one single particle and to one pixel of an eventcounting image. By accumulating event-counting images, a high spatial resolution image is obtained.

Here, using a two-microchannel plate (chevron) fluorescent screen assembly, the event-counting detection method is applied to low-energy electron imaging in a point projection microscope. Demonstration of $15 \mu \mathrm{m}$ resolution of the detector paves the way for working with a source-to-sample distance about one order of magnitude greater than previously achieved ${ }^{4,5,6}$. Moreover, the large number of detection events counted with electrons enables more accurate analysis of the detector response than previously achieved with neutrons ${ }^{1}$.

Note that the counting method used in transmission electron microscopes ${ }^{18}$ could not be applied here because electron energy is too small. Inversely, the technique reported here cannot be applied for high energy electrons because of the MCP limitations.

\section{Materials and methods}

The low-energy electron point projection microscope (figure 1 ) is a lensless setup ${ }^{19}$. An electron point source illuminates a perforated object (e.g. holey carbon film) which elastically scatters electron waves. The interference pattern of the reference wave (directly propagating from the source) and the scattered wave (propagating from the object) is produced on the fluorescent screen. The recorded pattern, showing a shadow surrounded by fringes, corresponds to the so-called hologram of the object $^{20}$. The numerical reconstruction of this hologram gives a magnified image of the object $^{21}$. The spatial resolution given by the microscope is obtained from the reconstructed image of the object.

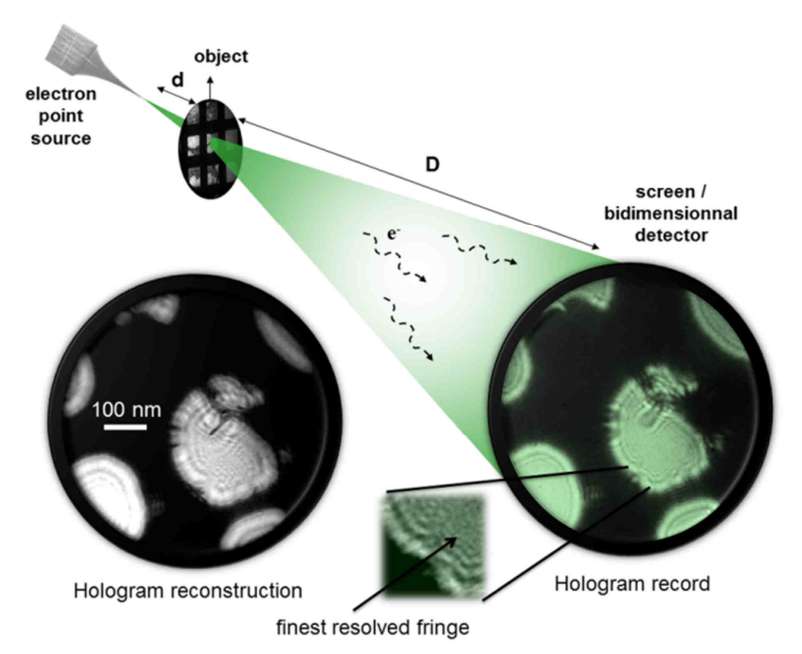

Figure 1: Low-energy electron point projection microscope. A CCD camera records the hologram produced via one microchannel plate in front of a fluorescent screen (the "bidimensionnal detector"). The numerical holographic reconstruction of this record gives the image of the object with a resolution of about $1 \mathrm{~nm}$ in the best cases.

Usually, the low-energy electron source is an ultra-sharp field emission $\mathrm{W}$ tip but noble-metalcoated $W$ tips $^{22}$, alloy tips ${ }^{23}$ or a carbon nanotube ${ }^{24}$ have also been used. Here, a more bulky recently developed source ${ }^{14}$ is used, based on the point emission from a mineral particle deposited on the apex of a $12 \mu \mathrm{m}$ diameter carbon fiber. The object is a holey carbon film which is sufficiently perforated to obtain a 
reference wave. The source-to-object distance $d$, is controlled by a piezoelectric actuator providing a distance control from $100 \mathrm{~nm}$ to $2.5 \mathrm{~cm}$. The screen is $56 \mathrm{~cm}$ away from the source. Because individual stains are required for the eventcounting method, the impact of one single electron at the entrance of the MCP has to produce a detectable stain on the screen. Therefore instead of a one-stage MCPfluorescent screen assembly, a two-stage MCP (Hamamatsu - F2221-21P) is used: the amplification given by the manufacturer is about $10^{6}$ that means up to a hundred times higher compare to one-stage. The optical microscopic view of the MCP (figure 2 ) shows a lattice hexagonal structure for the arrangement of the microchannels, whose diameter is only $12 \mu \mathrm{m}$. This suggests that electron impacts cannot be localized more accurately than this under our conditions (the minimum microchannel size available is $7 \mu \mathrm{m}$ ).

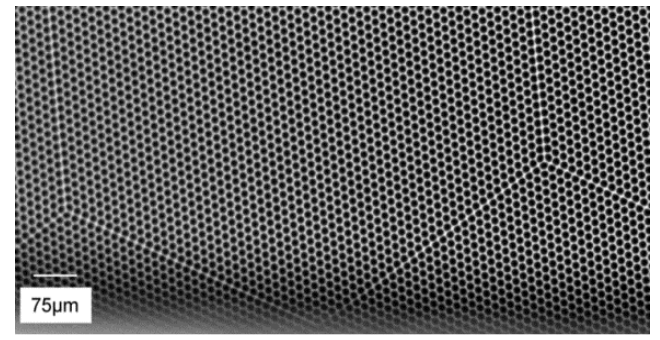

Figure 2: Optical microscopic view of the microchannel plate: large hexagonal bundles of channels are observable (side length $=450 \mu \mathrm{m}$ ). The lattice parameter of the microchannel hexagonal structure is about $a=14.6 \mu \mathrm{m}$ (channel diameter is $12 \mu \mathrm{m}$ ).

The whole screen diameter is $4 \mathrm{~cm}$. Images of the screen are recorded by a digital CMOS camera (Hamamatsu ORCA flash 4.0) providing a $2048 \times 2048$ pixel image (one pixel $=6.5 \mu \mathrm{m}$ ) in a minimum exposure time $t=3.05 \mathrm{~ms}$. The gray level dynamic available is either 8 or 16 bits encoded. An optical lens reduces the field of view recorded by the camera to a part of the screen, i.e. $2048 \times 2048$ pixels corresponds to $(12 \mathrm{~mm} \times 12 \mathrm{~mm})$ on the screen.
The event-counting method consists in collecting the centroid of each single stain. An individual stain is separately identified provided it is not too close to another. Thus electron intensity and time frame have to be adjusted in order to detect only a few stains per frame. With an exposure time of $10 \mathrm{~ms}$, we tune electron intensity to about $10^{-16} \mathrm{~A}$ on a $(3 \times 3) \mathrm{mm}^{2}$ part of the screen. This corresponds to a detection rate of about 5 electrons (stains) per (512x512) pixel frame. The detection rate could be higher than the one used but cannot give more than 500 stains per frame to be able to separate them. This gives a maximum intensity of about $10^{-14} \mathrm{~A}$ on a $(3 \times 3) \mathrm{mm}^{2}$.

The size and intensity of one stain depends on the amplification of the MCP. With a $2 \mathrm{kV}$ supply applied to the two-stage MCP and a $4 \mathrm{kV}$ acceleration voltage between the MCP exit and the screen, we observe stains with a typical full width at half-maximum (FWHM) of about 60 $\mu$ m. The signal-to-noise ratio $\left(S N R=\frac{\mu}{\sigma}\right)$ calculated by measuring the typical gray level of a stain $(\mu)$ and the standard gray level deviation of the whole recorded image $(\sigma)$ is about 10 .

The determination of centroids uses the plugin QuickPalm ${ }^{25}$ under ImageJ. QuickPALM is a set of program developments for PALM and STORM ("photoactivated localization microscopy" and "stochastic optical reconstruction microscopy"). A stain is detected depending on the Signal-toNoise ratio (SNR) and the typical full width at half maximum (FWHM). A first frame is recorded to determine the SNR and FWHM of a typical stain. These values are introduced as parameters in the program in order to assign the value ' 1 ' to one pixel in the center of one stain. Then a sequence of frames can be recorded and analyzed in order to produce multiple binary frames that are added together to create a counting image. Each frame is sent to the computer, recorded and then treated either immediately or at the end of the series. In the first case, recording time is lost between frames and in the second case, a computer memory of almost 1TB is required. To obtain representative information in the screen, 
considering the highest intensity $\left(10^{-14} \mathrm{~A}\right.$ on on a $(3 \times 3) \mathrm{mm}^{2}$ ) available with this method and the highest frame rate available with actual cameras, the whole screen can be completely lightened after minimum 500 frames then a minimum time of about $5 \mathrm{sec}$. With this electron rate, the minimum computer memory usable is about 4GB.

Therefore, we first record the whole image in analogical mode and second, we record only a zone of interest in counting mode. The analogical mode corresponds to the direct gray level image accumulated with the camera whereas the counting mode consists on adding the centroid single-event short frames. The emission intensity has to be tuned so as both to record an analogical image in a few seconds and to record the same image for hours (in conditions described here) in counting mode. In practice, in a low-energy electron microscope, the intensity increases with the electric field which increases with the source-object distance decrease and with the voltage applied to the source that gives the electron energy.

\section{2- Results and discussion}

To compare analogical and counting modes in terms of spatial resolution on the screen, the shadow of the edge of a carbon membrane is produced at low magnification in both modes for the same exposure time $t$ and the same electron dose. The cumulative images from $t=3 \mathrm{sec}$ to 3000 sec are shown in figure 3 . The first observation is that the image quickly appears in analogical mode. The second information is given from the final signal-to-noise ratio $\left(\frac{S}{N}\right)$ obtained in both conditions. It is measured with the ratio between the mean gray levels or the mean number of counts in the black part and in the lightened part of the analogical or counting images respectively. Here we find $\left(\frac{S}{N}\right)_{\text {anal }}=4.3$ and $\left(\frac{S}{R}\right)_{\text {count }}=7.7$. This signal-to-noise ratio is always better in counting mode but can be much higher if the SNR chosen to detect stains is much higher. For example, figure 3 was constructed with a SNR=20 if we choose a $S N R=30$ then the final signal-to-noise ratio is about $\left(\frac{S}{R}\right)_{\text {count }}=16$ and the bright spots observables in figure 3 decrease.

Finally, from figure 3 we can measure that the spatial resolution is better in counting mode. This is illustrated by the intensity profile through the edge of the shadow in figure 4.

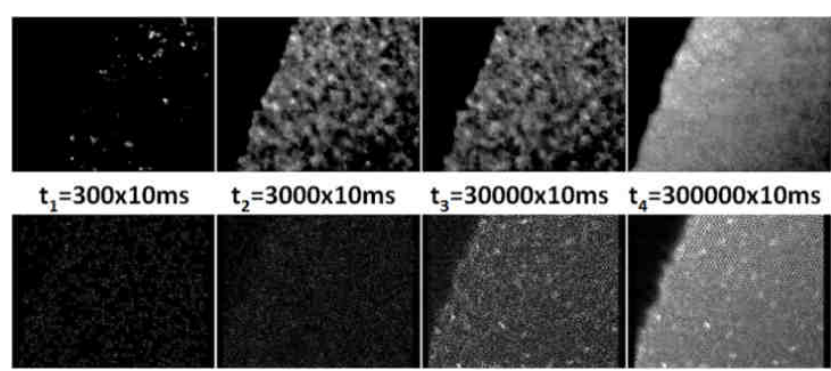

Figure 3: Construction of cumulative analogical and counting records. The upper line corresponds to the analogical images with a gray level representation; the lower line corresponds to the counting images with calculation of stain centroids and the sum of each binary image. $\mathrm{I}=8.5 \times 10^{-17} \mathrm{~A},(3 \times 3) \mathrm{mm}^{2}$ area on the screen; $2 \mathrm{kV}$ applied to the MCPs, electron energy of about $500 \mathrm{eV}$.

Profiles are fitted by an error function (convolution of a step function assumed for the shadow and a Gaussian response of standard error $\sigma$ assumed for the detection). In analogical mode, the fit closely follows the recorded profile and corresponds to $\sigma=95 \mu \mathrm{m}$ (coefficient of determination: $R^{2}=0.99$ ). In counting mode, $\sigma=18 \mu \mathrm{m}$ although the recorded profile fluctuates a lot around the fit (coefficient of determination: $R^{2}=0.08$ ). A close look at the profile indicates that detection events are counted every $15 \mu \mathrm{m}$. This suggests that detection events appear only at the microchannel locations, none being counted in the surrounding area. At the edge of the counting profile the signal changes abruptly, suggesting that its position is indeed defined by the size of the channel. 


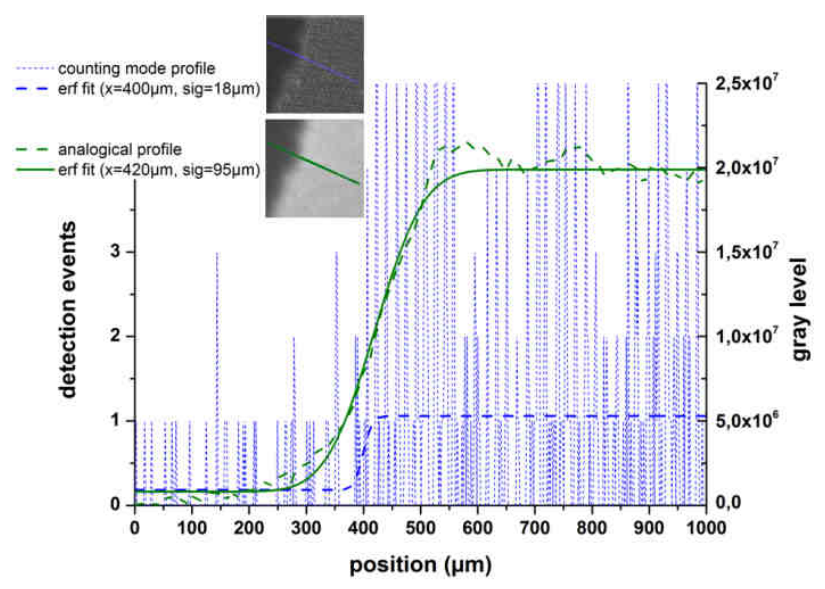

Figure 4: Signal profiles along the same line of analogical and counting images of the same edge.

Measuring the spatial resolution of the detector by its response to a sharp edge image is not the sole method possible. Because an electron impact can only be detected when it occurs inside a channel (as suggested by the counting record of figure 4), the arrangement of these channels can be determined simply by looking at the bright part of the image where the average intensity is constant. Such a counting image is shown in figure 5 . Several observations can be made: first, the microchannel bundle boundaries visible in optical microscopy are recognizable; second, detection spots appear along lines; third, the intensity of these spots is highly variable. Note that the lines (figure 6a) cross the boundaries of the bundles without changing direction. These lines look like the reticular lines of the 2D lattice of the microchannels observed in optical microscopy where a distance between adjacent channel centers $a=14.6 \mu \mathrm{m}$ is measured. However because of the change in intensity of the spots, we perform FFT and amplitude analysis of the image.

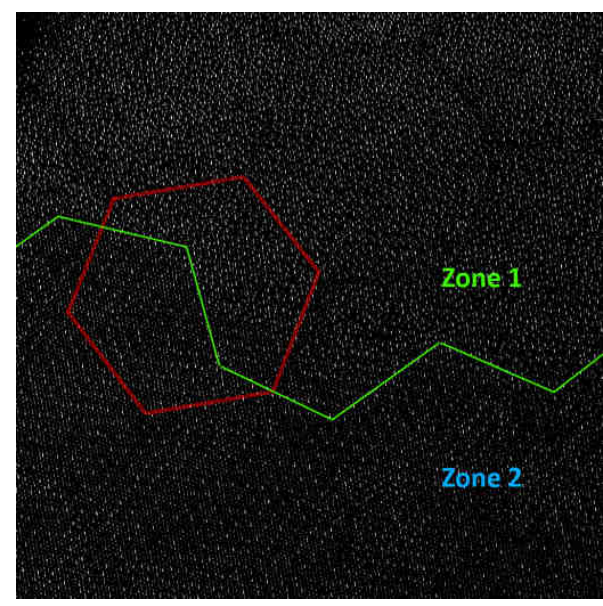

Figure 5: Counting image in a uniformly lit part of the screen: detection is localized (bright spots), a microchannel bundle is located by the hexagonal red line. A polygonal boundary (green line) between two zones (zone 1 and 2) of different average intensity is visible.

This FFT (figure 6b) shows a hexagonal structure with a distance between reticular planes of $d_{r}=a \frac{\sqrt{3}}{2}$, with $a$ the lattice parameter. This result already obtained with neutrons ${ }^{1}$ confirms the previous observation at an edge. This hexagonal structure in the FFT appears with relatively poor statistical sampling (i.e. with widely fluctuating intensity of the spots), which means that the structure of the MCP suggests that of a hexagonal bi-dimensional crystal. With larger statistical sampling ( $>500.000$ detection events on a $512 \times 512$ pixel image), a hexagonal superstructure appears in the FFT (in blue in the FFT, figure 6c). This superstructure is rotated by $\alpha=(15 \pm 1)^{\circ}$ from the microchannel hexagonal lattice. Its lattice parameter $s$ presents a dimension ratio of $\frac{s}{a}=3.6$ relative to the lattice parameter $a$ of the hexagonal microchannel structure. While intensity is not uniform in the two zones identified in figure 5 , this superstructure is the same in both zones. 


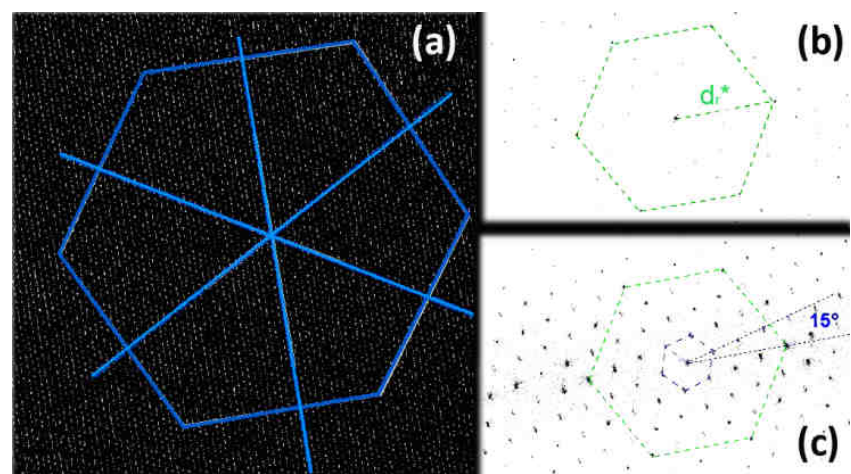

Figure 6: (a) close-up of a hexagonal bundle with lines indicating the main alignments of detection sites; (b) FFT of the screen image for $t_{2}=$ $3000 \times 10 \mathrm{~ms}$ : the lattice parameter measurable in the FFT is $a=14.6 \mu \mathrm{m}$ (c) FFT of the screen image for $t_{4}=300000 \times 10 \mathrm{~ms}$ : a superstructure appears (blue) $(\mathrm{s}=46.5 \mu \mathrm{m})$. Its FFT is $(15 \pm 1)^{\circ}$ rotated from the main channel structure.

This superstructure was hardly visible in the Tremsin et al. ${ }^{1}$ records due to poorer statistical sampling than in the present work. This means that their interpretation of the FFT result cannot satisfactorily explain the whole superstructure. The geometry of the chevron mounts of the microchannels was held responsible for a "nonsymmetric hexagonal pattern", which is not sufficient to explain a superstructure in the FFT. We show hereafter that the superstructure likely results from a Moiré effect induced by the rotation of one MCP with respect to the other in the assembly. Calculations to determine the different rotation angles for which a superstructure appears are presented in the appendix. The channel network is considered as a perfect hexagonal lattice. The shortest superstructures (with lattice parameter $s$ such that $\frac{s}{a}<6$ ) appear for only 5 rotation angles: $\beta=21.8^{\circ}, 27.8^{\circ}, 13.2^{\circ}, 17.9^{\circ}$ and $9.4^{\circ}$ with the respective dimension ratio $\frac{s}{a}=2.64,3.60,4.36$, $5.57,6.08$. Clearly the present work corresponds to the second case, since the corresponding dimension ratio of 3.6 is in close agreement with the experimental result of figure $6 \mathrm{c}$. A simulation of the FFT created from a $27.8^{\circ}$ rotation between MCPs is presented in figure 7. In this simulation the FFT is applied to an image built by superimposing one part of the optical microscopy view of the MCP (figure 2 ) on an identical part of the view which is rotated $27.8^{\circ}$. This FFT shows a rotation angle in the Fourier space: $\alpha=\frac{\beta}{2}=13.9^{\circ}$ that appears quite similar to the experimental pattern of figure $6 \mathrm{c}$.
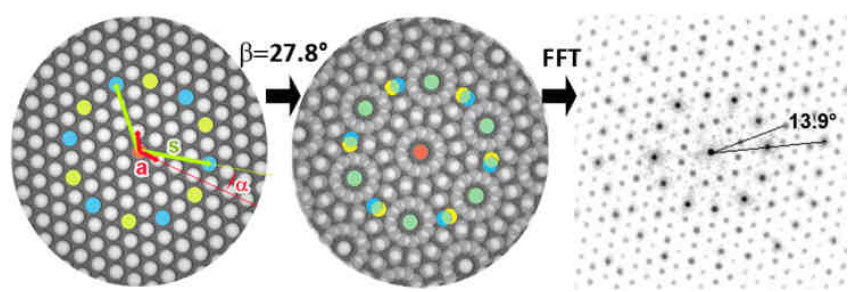

Figure 7: Simulation of rotation between MCPs and the related FFT. A rotation of $27.8^{\circ}$ between MCPs leads to a FFT giving a dimension ratio between the microchannel network and the superstructure of $\frac{s}{a}=3.6$ and an angle between them of $\alpha=13.9^{\circ}$.

Another effect results from the relative positioning of the two plates. Looking at the stain shape in original data records, it appears that three different kinds of stain are observable (figure 8a): two are circular with diameters of about $40 \mu \mathrm{m}$ and $60 \mu \mathrm{m}$ and one is elliptical, about $60 \mu \mathrm{m}$ long and about $40 \mu \mathrm{m}$ wide. Although the observed stain is much bigger than the channel diameter, it seems that the corresponding kind of site depends on the number of channels of the second MCP that overlaps one channel of the first plate. Figure $8 \mathrm{~b}$ shows the image built by superimposing an optical microscopy image of the MCP with the same image rotated $27.8^{\circ}$ : the three kinds of site are clearly identified in this view. Analysis of the size distribution of stains (figure 8 ) indicates two characteristic sizes: 40 and $60 \mu \mathrm{m}$. Length and width are reported together so these two peaks are characteristic of the three kinds of site. 


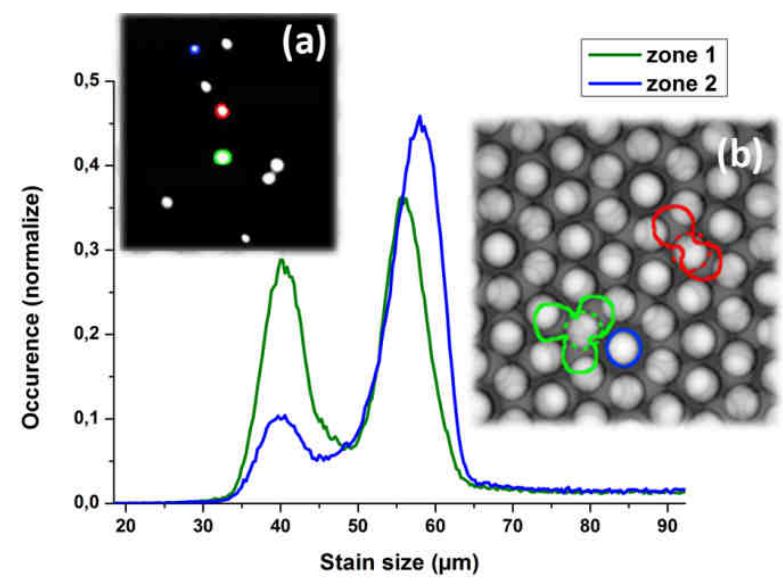

Figure 8: Histogram of stain size distribution in zones 1 and 2 of figure 5 (from original data) (a) shape of detected stains showing three kinds of site: two circular (blue and green) and one elliptical (red) - (b) sum of the microscope view of the MCP and the same view rotated (here $\left.27.8^{\circ}\right)$. One channel from the first MCP overlaps with one (blue), two (red) or three (green) channels of the second MCP.

However, a different distribution is found in zone 1 and zone 2 as defined in figure 5 . Clearly the ratio of $60 \mu \mathrm{m}$ stains to $40 \mu \mathrm{m}$ stains is much higher in zone 2 . To see how this can be related to the stacking of the plates, we examine a small random translation in addition to the $28^{\circ}$

rotation between the two plates. This is shown in figure 9. The FFTs produced by these two situations are the same, which may explain the variable intensities observed in zones 1 and 2 in figure 5 .

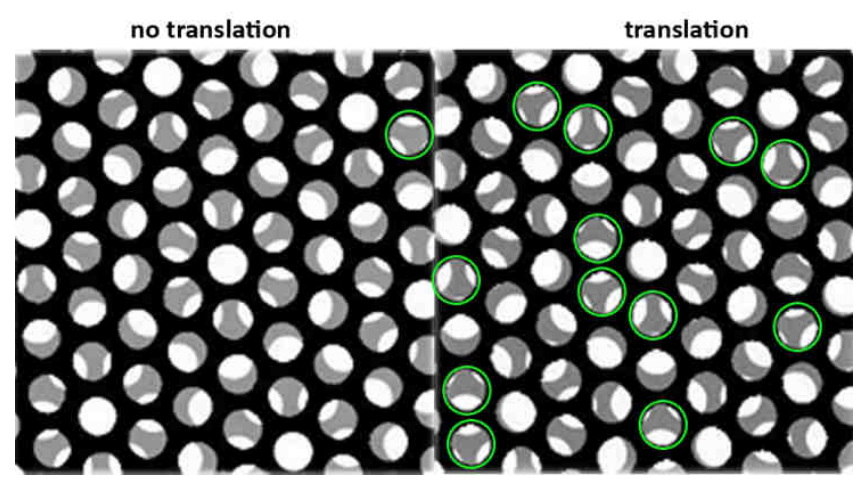

Figure 9: Illustration of the different kinds of site obtained with and without translation. The white part in channels defines the number of sites impinging on the second MCP. Without translation there are few sites where one channel overlaps with 3 channels (surrounded in green line), a small translation increases this number significantly ( $8 \mu \mathrm{m}$ in one direction), and the translation does not change the FFT.

\section{3- Conclusion}

Two methods were used to determine the resolution of an imaging detector composed of a two-stage MCP fluorescent screen assembly used in a counting mode in a low-energy electron projection microscope. The first method is based on viewing the edge of a carbon membrane, the second method is based on a uniform electron illumination of the assembly. Both methods show that the response of the detector is described by a network of detection sites that closely reflects the geometrical structure of the microchannel packing. This leads to a spatial resolution of $\sim 15 \mu \mathrm{m}$ which is the shortest distance between the centers of two microchannels. While the overall response reflects the hexagonal structure of the microchannel packing in one plate, we show that the local response of the detector varies because of the relative positioning of the two MCPs (rotation and translation).

In a low-energy electron point projection microscope the enhanced resolution of the detection, from $100 \mu \mathrm{m}$ (previously obtained by conventional recording of the image on the screen) to $15 \mu \mathrm{m}$ distance between microchannels (in the present work, but possibly $10 \mu \mathrm{m}$ with smaller diameter microchannels), paves the way to working with a larger sourceto-sample distance (about one order of magnitude greater than previously achieved). This widens the source property requirements for subnanometer resolution imaging. It would be worth implementing immediate treatment of detection events inside the camera (determination of the centroid of stains) before transfer to the computer to improve image acquisition time.

\section{Acknowledgment}

The authors would like to thank Marjorie Sweetko for improving the English of this article and Yannick Boursier from CPPM for constructive 
discussions on the statistically representative information. 


\section{Appendix}

The calculation consists in the identification of rotations of two hexagonal lattices leading to a periodic structure. Because of the bijection between a plane and the complex number space, positions of nodes of the lattice are identified by complex numbers.

Here $(\vec{a}, \vec{b})$ are the base vectors of the first MCP with: $\vec{b}=\vec{a} e^{i \theta}$ with $\left(\theta=120^{\circ}\right)$.

$(\vec{u}, \vec{v})$ are the base vectors of the second MCP with: $\vec{u}=\vec{a} e^{i \alpha}$ and $\vec{v}=\vec{a} e^{i(\theta+\alpha)}$ with $\alpha$ the rotation angle between MCPs.

In a network, the position of the repetitive structure can be expressed with indexes along the base vectors. A superstructure appears if the two base vectors of the two MCPs coincide. Then the base vectors of the superstructure $(\vec{s}, \vec{t})$ can be expressed as:

$$
\begin{gathered}
\vec{s}=n \vec{a}+m \vec{b}=k \vec{u}+l \vec{v} \text { where } \mathrm{n}, \mathrm{m}, \mathrm{k}, \mathrm{I} \text { are } \\
\text { integers. }
\end{gathered}
$$

The distance ratio between the structure and the superstructure is:

$$
\frac{|\vec{s}|}{|\vec{a}|}=\sqrt{n^{2}+m^{2}+n m}=\sqrt{k^{2}+l^{2}+k l}
$$

And the correspondence of scalar and vector products gives:

\begin{tabular}{|c|c|c|c|c|c|}
\hline $\begin{array}{l}\text { s/a frotation ansle } \\
\text { between MCPSy) }\end{array}$ & $2.64(21.87)$ & $3.6(27.87)$ & $4.36(13.27)$ & $5.57(17.97)$ & $6.08\left(9.4^{4}\right)$ \\
\hline Before rotation & & & & & \\
\hline After rotation & & & & & \\
\hline Simulation ffr & 4 & 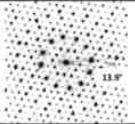 & $\begin{array}{l}x+4 \\
4 x+4\end{array}$ & 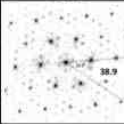 & 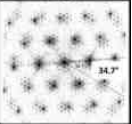 \\
\hline
\end{tabular}

$$
\begin{gathered}
n-\frac{1}{2} m=\left(k-\frac{l}{2}\right) \cos \beta-\frac{\sqrt{3}}{2} l \sin \beta \\
\frac{\sqrt{3}}{2} m=\left(k-\frac{l}{2}\right) \sin \beta+\frac{\sqrt{3}}{2} l \cos \beta
\end{gathered}
$$

Solving this equation for $\left|\frac{s}{a}\right|<6$ leads to:

${ }^{1}$ A.S. Tremsin, J.V. Vallerga, J.B. McPhate, O.H.W. Siegmund, W.B. Feller, L. Crow, and R.G. Cooper, Nuclear Instruments and Methods in Physics Research A 592, 374 (2008)
${ }^{2}$ W. Stocker, HW Fink and R. Morin, Ultramicroscopy 31, 379 (1989)

${ }^{3}$ E. Steinwand , J.-N.Longchamp, H.-W.Fink, Ultramicroscopy 110, 1148 (2010)

J. Bardon, A. Degiovanni, V. Georges, and R. Morin, Ultramicroscopy 92, 133 (2002)

${ }^{5}$ A. Eisele, B. Völkel, M. Grunze, A. Gölzhäuser, Zeitschrift für Physikalische Chemie 222(5-6), 779 (2008)

${ }^{6}$ J.N. Longchamp, S. Rauschenbach, S. Abb, C. Escher, T. Latychevskaia, K. Kern, HW Fink, Proceedings of the nat. academy. of Sciences of USA $114(7), 1474$ (2017)

${ }^{7}$ V. Georges, J. Bardon, A. Degiovanni, and R. Morin, Ultramicroscopy 90, 33 (2001)

${ }^{8}$ Beyer A , Goelzhaeuser A , Journal of Physics- Condensed Matter 22, 343001, (2010)

9 Hwang IS, Chang CC, Lu CH, Liu SC, Chang YC, Lee TK, Jeng HT, Kuo HS, Lin CY, Chang CS, Tsong TT, New Journ. Phys. 15 (2013)

${ }^{10}$ M. Prigent, P. Morin, J. of Phys. D- Applied Physics 34(8), 1167 (2001)

${ }^{11}$ M. Prigent, P. Morin, J. of Microscopy - Oxford (199), 197 (2000)

${ }^{12}$ R. Morin, Microscopy Microanalysis Microstructures 5(4-6), 501 (1994)

${ }^{13}$ T. Latychevskaia, Ultramicroscopy 175, 121 (2017)

${ }^{14}$ E. Salançon, R. Daineche, O. Grauby, and R. Morin, JVST B 33, 030601 (2015)

${ }^{15}$ N. Yasuda, H. Suzuki and T. Katafuchi, Radiol. Phys. Technol. 9, 88 (2016)

${ }^{16}$ O. Siegmund, A. Tremsin, J. Vallerga and J. McPhate, Nucl Instrum Methods Phys Res A. 610(1), 118 (2009)

17 J. Vallerga, A. Tremsin, R. Raffanti and O. Siegmund, Nucl Instrum Methods Phys Res A.; 633(S1), S255 (2011)

${ }^{18}$ LC Gontard, G Moldovan, R Carmona-Galan., C Lin, Al Kirkland, Microscopy 63(2), 119-130 (2014)

${ }^{19}$ W. Stocker, HW Fink and R. Morin, Ultramicroscopy 31, 379 (1989)

${ }^{20}$ H. W. Fink, W. Stocker, H. Schmid, Phys. Rev. Lett. 65, 1204 (1990)

${ }^{21}$ H.J. Kreuzer, K. Nakamura, A. Wierzbicki, H.W. Fink, H. Schmid, Ultramicroscopy 45, 381 (1992)

${ }^{22}$ Chang CC, Kuo HS, Hwang IS, Tsong TT, Nanotechnology 20(11), 115401 (2009)

${ }^{23}$ K. Nomura, T. Nagao, BL. Cho, H. Katsuda, T. Matsumura, C. Oshima, JVST B 27(6), 2432 (2009)

${ }^{24} \mathrm{~N}$. de Jonge, Jour. Appl. Phys. 95(2), 673 (2004)

${ }^{25}$ R. Henriques, M Lelek, E.F. Fornasiero, F. Valtorta, C. Zimmer, M.M. Mhlanga, Nature Method 7, 340 (2010) 